\title{
BMC Nutrition reviewer acknowledgement, 2015
}

James Mockridge

\section{Contributing reviewers}

The editors of BMC Nutrition would like to thank all our reviewers who have contributed to the journal in Volume 1 (2015).

\author{
Ammar Ashor \\ UK \\ Adam Rahman \\ Canada \\ Grant Aaron \\ Switzerland \\ James B. Adams \\ USA
}

Abdul Momin Rizwan Ahmad

Pakistan

Diane Allen-Gipson

USA

Sergio Amarri

Italy

Ruopeng An

USA

Emmanuel Anígilájé

Nigeria

Luis Antunes

Portugal

Andrea Apicella

Italy

Melita Avdagovska

Canada

\section{Mahmoud Bahmani}

Iran

Christine Baldwin

UK

\author{
Terri Ballard \\ Italy
}

Robert Bandsma

Canada

Samira Barcelos

Brazil

Tyler Barker

USA

Eliane Barroso

Brazil

Benjamin Becerra

USA

Kathryn Beck

New Zealand

John Bertram

Australia

Samiran Bisai

India

Sonia Blaney

Central African Republic

Elaine Borghi

Switzerland

Fernanda Busnello

Brazil

Nguyen Huu Chau Duc

Vietnam

Mariana Chilton

USA
Hyo Geun Choi

Democratic People's Republic of Korea

Eva Clausson

Sweden

Estefania Custodio

Spain

Valesca D'Alba

Brazil

Leonie Dapi Nzefa

Sweden

Subal Das

India

Ian Davies

UK

Peter Davies

Australia

Arnaud De Luca

France

Vanessa De Mello Laaksonen

Finland

Abraham Degarege

Ethiopia

Diane Dellavalle

USA

Jennifer Di Noia

USA

Ming Ding

USA

Correspondence: james.mockridge@biomedcentral.com

BioMed Central, Floor 6, 236 Gray's Inn Road, London WC1X 8HB, UK 
Orianne Dumas

France

Yvonne L. Eaglehouse

USA

Mehmet Emin Kuyumcu

Turkey

Ingunn Engebretsen

Norway

Flavia Fernandes

Brazil

Lauren Fiechtner

USA

Vinncenzo Fierro

Italy

Robert Fungo

Uganda

Cheryl Gammon

New Zealand

Eduardo Garcia-Fuentes

Spain

Michael Georgoulis

Greece

Maria Gianni'

Italy

Heather Godwin

UK

Su Golder

UK

Adrian Gombart

USA

Yevgeniy Goryakin

UK

Anna Grandone

Italy

Ornella Guardamagna

Italy

Honghui Guo

China

Neha Gupta

India

Roberta Hack Mendes

Brazil

Mary Hearst

USA
Ditte Hobbs

UK

Stephen Hodgins

USA

Andrew Holwerda

Netherlands

Susan Horton

Canada

Steffen Husby

Denmark

Koen Huysentruyt

Belgium

Fumiaki Imamura

UK

Shevin Jacob

USA

Chris Jolly

USA

Lamis Jomaa

Lebanon

Andrew Jones

USA

Melanie Jordan

USA

Janine M. Jurkowski

USA

Haji Kedir

Ethiopia

Marko Kerac

UK

Atika Khalaf

Sweden

Dorcus Kigaru

Kenya

Ruth Kimokoti

USA

Stanislaw Klek

Poland

Tomasz Kostka

Poland

Katharina Kovacs Burns

Canada

Herculina Salome Kruger

South Africa
Praveen Kumar

India

Anton Kunst

Netherlands

Elisabeth Kylberg

Sweden

Molly Lamb

USA

Edwige Landais

France

Rebecca Lander

USA

Lisa Langsetmo

Canada

Jeannine Lawrence

USA

Sophie La Vincente

Australia

Jean Yves Lefrant

France

Shannon Lennon-Edwards USA

Bess Lewis

USA

Duo Li

China

Jun Liang

China

Karen Lindsay

Ireland

Katherine Mary Livingstone Australia

Meghan Longacre

USA

Barbara Lourenco

Brazil

Jacqueline Lowdon

UK

Yiyi Ma

USA

Una Macintyre

South Africa

Kenneth Maes

USA 
Ken Maleta

Malawi

Wan Manan

Malaysia

Mark Manary

USA

Subha Mani

USA

Sandra Marinho

Brazil

Elisabetta Marini

Italy

Luise Marino

UK

Lucia Marseglia

Italy

Maria Carmen Martinez Cuesta Spain

Pierluigi Marzuillo

Italy

Jessie-Lee McIsaac

Canada

Rosan Meyer

UK

Brandy-Joe Milliron

USA

Erik Millstone

UK

Parvin Mirmiran

Iran

Nitish Mondal

India

Daniel Moore

Canada

Wyn Morgan

UK

Malay Mridha

USA

Owen Mtambo

Namibia

Beverly Muhlhausler

Australia

Emma Nilsson

Sweden
Rashed Noor

Bangladesh

Faryle Nothwehr

USA

Paulina Nowicka

Sweden

Tinuade Ogunlesi

Nigeria

Wilna Oldewage-Theron USA

Leticia Olivera

Mexico

Augustine Omoigberale

Nigeria

Jennifer Osei

South Africa

Sangshin Park

USA

Alexandr Parlesak

Denmark

Sant-Rayn Pasricha

Australia

Mitesh Patel

USA

Marcella Pedullà

Italy

Noel Peretti

France

Etienne Phipps

USA

Catherine Pound

Canada

Danijela Ristic-Medic

Serbia

Khaled Saad

Egypt

Mahama Saaka

Ghana

Ayesha Saeed

Pakistan

Hibbah Saeed

UK

Ambika Satija

USA
Robert Scragg

New Zealand

Hemant Shewade India

Flávia Silva

Brazil

Justin Silver

Israel

Piia Simonen

Finland

Hee-Jung Song

USA

Ranjani Starr

USA

Welma Stonehouse

Australia

Anne Sumner

USA

Guang Sun

Canada

Bruno Sunguya

Tanzania

Brian Symon

Australia

Alice Tang

USA

Valerie Tarasuk

Lebanon

Ali Tarighat-Esfanjani Iran

Francis Tayie

USA

Norman Temple

Canada

Chantale Tippett

Canada

Elke Trautwein

Netherlands

Guglielmo Trovato Italy

Josep A. Tur Spain

Emma Tzioumis

USA 
Stephan Van Vliet

USA

Fiona Warren

UK

Daniel West

USA

Maxine Whittaker

Australia

Pattanee Winichagoon

Thailand

Thomas Wolever

Canada

Jyh Eiin Wong

Malaysia
Julie Woods

Australia

Charlotte Wright

USA

Juan Wu

USA

David Ht Yen

Taiwan

Akar Yilmaz

Turkey

Zhi Yu

USA

David Zhao

China
Yimin Zhao

China

Ju-Sheng Zheng China

Qianling Zhuang

China

Canan Ziylan

Netherlands

Francis Zotor

Ghana 